\title{
Contents, Vol. 38, 1917
}

Inhalts-Verzeichnis.

Origraal-Arbeiten. S S $\beta$ ite

Berg, F., Ein Fall von latentem Nystagmus 164

Birch-Hírschfeld, A., Weitere Untersuchungen über die Nacht-

blindheit im Kriege 57

Bruckner, A., Zur Frage der Emigration einkerniger Blutzellen,

zugleich ein Beitrag zur pathologischen Anatomie der

sympathisierenden Entzớndong ohne sympathisehe Oph-

$\mathrm{tl} \cdot$ almie 139

-.-- Zur Frage der Lokalisation des Kontrastes und ver-

wandter Erscheinungen in der Sehsinnsubstanz .... 1 Carsten, P., Ein weiterer Fall von

Cysticercus subretinalig.

(Hierzu Taf. VII- X) 296

Deutschmann, B., Zur Frage des traumatischen Glaukoms.

(Erwidening an Peters)

209

Doesschate, G. ten, Beitrag zur Kenntnis der Bindegewebs-

bildungen an der Sehnervenpapille des Menschen. (Hierzu

3 Abb. auf Taf. I) 197

F'ritsch, 11., Zur Kasuistik des Exophthalmus pulsans ... 186 Gilbert, W., Die syphilitische

Lymphomatose der Bindebaut

mit Membranbildong. (Hierzu Fig. 4 auf Taf. I) . 152 v. d,, Hoeve, J. Röntgenuntersuchung bßi

Tumoren der Orbita 156

Jánô, A., Lenticonus posterior . .

Kniisel, 0., Arecolin mit besonderer Berücksichtigung seiner

Wirkung auf das glaukomatöse Auge 15

Koeppe, L., Über Hemeralopie als Folge einer eigentümlichen

und wahrscheinlich angeborenen Linsenveränderung . . 89 -,- Über Heilung zweier Fälle von

Ulcus rodens corneae

durcb Tuberkvlininjektionen nebst Bemerkungen über die

mikroskop.-anatom. Untersuchung eines dritten Falles

(Hierzu Tal. VI) 301

Löwenstein, A., Zungenförmiger Lappen mit seitlichen Ent-

spannungsnähten, bei der Kuhntschen Bindehautplasl ik. 190 Ohm, J., Über den Einfluß des

zweiä $\alpha$ gigen Sehens auf den

Nystagmus 269

Peters, A., Zur Frage des traumatischen Glaukoms. (Er-

widerung an Deutschma,nn) . 202

Piehler, A., Korrektur einer schwachen Myopie durch ein rotes

Planglas $\quad 174$ 
- -,- Zur Kenntnis des Blitzstares . 105

- IV -

Scite

Pichler, A., Zur Naht zarter Epidermislappen ...... 202

Schürmann, J., Weitere Untersuchungen über die Linsen-chagrinierung, sowie über Häufigkeit und anatomiscb.es Verhalten der Chagrinkugeln und ihre Beziehungen zur

Katarakt 42

Síargardt, K., Phthiriasis der Lider mit Follikularkatarrb . 288

SziJclai, P., Zur Frage des Entropium congenitum 103

Waldmann, B., Kriegserfahrungen und Studie auf dem Gebiete

der Opbthalmo-Blennorrhoea adultorum 177

Wolff, H., Vereinfachte Erörterung über Skiaskopie. Nebsteiner Übersicht über 393

Untersuchungen. HierzuTat II-V) 318

Bach, H., Erwiderung auf die „Bemerkung” des Herrn San.-Rates Dr. Fr. Schanz- $\Omega$ xesden in Heft 3-4 der Zeitschrift

für Augenheilkunde. Bd. XXXVII 107

Theodor Leber $\uparrow$. Xacbruf von Dr. E. Seidel in Heidelberg 258

Carl Mellinger $\uparrow$. Von Dr. A. Erb in Lugano 128

Bericht des Ausschusses zur Schaffung von Richtmaßen fürBrillengläser

undBrillenglasfassungen. Von Dr. 0. Henker, in Jena 212

Berichte über deutsche ophthalmologische Literatur.

Dioptrik und Anomalien der Refraktion und Akkommod, ation

(1916). Von Prof. Dr. M. Salzmann in Graz .... 108

Optik. Von Dr. Hugo Wolff in Berlin 220

Spezielle Pathologie und Therapie (1916. I. Semester). Von

San.-Rat Dr. Schroder in Gera 232, 360

Physiologie des Gesichtssinnes und der Aagenbewegungen ' (1916. II. Sem.). Von Prof. Dr. H.

Köllner in Würzburg 120

Berichte üher die auslänßische ophthalmologische Literatur.

Bericht über die holländische ophthalmoiogische Literatur(1917. I. Sem.). Von Prof. Dr. W.

Koßter-Ozn in Leidenund Dr. O. J. Schoute in Amsterdam 369

Therapeutische Umschau . . 132,262,374

Literatur-Verzeichnis 136,264,379

Buchanzeigen * 133

personalien .. 138383

S. Sachregister $\quad .383$

Namenregistei $\lambda \quad 386$ 